\title{
Entropy linear response theory with non-Markovian bath
}

\section{Yu Chen}

Graduate School of China Academy of Engineering Physics, P.R. China

E-mail: ychen@gscaep.ac.cn

ABSTRACT: We developed a perturbative calculation for entropy dynamics, which considers a sudden coupling between a system and a bath. The theory we developed can work in a general environment without Markovian approximation. A perturbative formula is given for bosonic environments and fermionic environments, respectively. We find the Rényi entropy response is only related to the spectral functions of the system and the environment, together with a statistical kernel distribution function. We find a $t^{2}$ growth/decay in the short time limit and a linear $t$ growth/decay in a longer time scale for the second Rényi entropy response. A non-monotonic behavior of Rényi entropy for fermionic systems is found to be quite general when the environmental temperature is the lower one. A Fourier's law in heat transport is obtained when two systems' temperatures are close to each other. A consistency check is made for Sachdev-Ye-Kitaev model coupling to free fermions, a Page curve alike dynamics is found in a process dual to black hole evaporation. An oscillation of Rényi entropy is found for an environment with a gapped spectrum.

KEYwords: 1/N Expansion, Holography and condensed matter physics (AdS/CMT), Quantum Dissipative Systems

ArXiv EPrint: 2012.00223 


\section{Contents}

1 Introduction 1

2 General entropy response theory $\quad 2$

2.1 General features for bosonic systems

2.2 General features for fermionic systems 8

3 Renyi entropy response in SYK model $\quad 9$

3.1 Heating and evaporation with Markovian-like environment 9

$\begin{array}{ll}3.2 & \text { Heating and evaporation with non-Markovian environment }\end{array}$

4 Conclusion $\quad 13$

\section{Introduction}

Linear response theory (LRT) is essential for studying quantum matters. It lays the foundation for physical interpretations of the dynamical response after a perturbative probe to the system [1]. LRT is widely used in all kinds of condensed matter experiments, cold atoms experiments, such as APRES, conductivity, neutron scattering, etc. In the original linear response theory, the dynamics of physical observables are considered in response to external sources. These perturbations are hermitian couplings to the systems. However, when we consider a more general case for dissipative couplings, a new response theory is needed and established as a Non-hermitian linear response theory (NHLRT) [2]. A relation between dissipative dynamics [3] and the correlation function in the initial equilibrium state can be then established with the help of NHLRT.

However, previous response theories are mostly focused on physical observables, entropies response theories for a general environment is still not fully established. In this paper, we are going to establish a general perturbative response theory for entropy with a generic bath. Nowadays, thermal entropies, as well as entanglement entropies have become increasingly important in studying eigenstate thermalization [4-7], information scrambling [8, 9], and quantum gravity [10]. It is in the core of holographic duality [11] which bridges the conformal field theory and the gravity through Ryu-Takayanagi formula [12-14]. In addition to these theoretical interests, new technological advances are also achieved recently in experimental measurements of the Rényi entropy (RE) [15-17]. All these theoretical and experimental studies make a general response theory for entropy a necessity.

Following the spirit of LRT, by taking the system-environment coupling as a perturbation, we can establish a linear response theory of Rényi entropy for a general system subject to a sudden coupling with an environment. Here we assume the bath is non-Markovian. 
The term non-Markovian means the temperature and spectrum of the environment can be arbitrary. Now we summarize our results as follows. We find that to the lowest order of coupling strength, the Rényi entropy response is only related to the spectral functions of the system and the environment, as well as a statistical kernel function. General properties of bosonic systems and fermionic systems are discussed and we predict a short time quadratic $t$ growth (decay) and long time linear $t$ growth (decay).

Further, we apply the linear response theory to Sachdev-Ye-Kitaev (SYK) model [20-24] with a free fermion bath at different temperatures. SYK model is recently discovered as an example of holographic duality [24-26]. It is dual to a Jackiw-Teitelboim (JT) gravity [27, 28] in $\mathrm{AdS}_{2}$ spacetime [23, 24]. Since the SYK model is solvable in large $\mathrm{N}$ limit, quite a few results for the SYK model's entropy dynamics are obtained for various SYK-like models [29-37] and for the Maldacena-Qi model [38] which is dual to a traversable wormhole. These entropy dynamics are also calculated on gravity side [39-43]. Here we try to ask, to what extent could our perturbative theory apply in a general case. Can we recover the Page curve in " black hole " evaporation $[44,45]$ where SYK model's initial temperature is higher than the environmental temperature? A Page curve is an entanglement entropy curve of the environment which follows a linear decay after initial linear growth. Even though we are calculating the black hole's entropy, still, we wonder if we can see signals for the Page curve. Eventually, we observed this Page curve alike behavior in long-time dynamics. Further, we change a gapless fermion bath to a gapped fermion bath so that the environmental spectrum changes dramatically. An interesting phenomenon is observed for RE oscillation at a special temperature window. It seems that the entangled information can travel back and forth between the system and the bath in this situation, which is quite curious.

Our paper is organized as follows. In the second section, we establish the general linear response theory for RE of the system. Then we discuss the general properties of RE dynamics for bosonic systems and fermionic systems. In the third section, we apply our theory to the SYK model with a non-Markovian bath. We change the temperature and the spectrum of the environment to calculate the RE response. Finally, we summarize our results in the conclusion section.

\section{General entropy response theory}

Here we consider a general situation for a sudden coupling between a system and a bath. Before the interaction, the Hamiltonian is $\hat{H}_{0}=\hat{H}_{\mathrm{S}}+\hat{H}_{\mathrm{E}}$, where $\hat{H}_{\mathrm{S}}$ and $\hat{H}_{\mathrm{E}}$ are Hamiltonian of the system and the environment respectively. After the coupling, the Hamiltonian becomes

$$
\hat{H}=\hat{H}_{\mathrm{S}}+\hat{H}_{\mathrm{E}}+\hat{V} .
$$

Here $\hat{V}=g \sum_{j}\left(\hat{O}_{j}^{\dagger} \hat{\xi}_{j}+\hat{\xi}_{j}^{\dagger} \hat{O}_{j}\right)$, where $\hat{O}_{j}$ is an operator acting on the system and $\hat{\xi}_{j}$ is an operator in the environment. $j$ is the index of the operator. We can find out that the 
system's density operator evolutes like follows

$$
\begin{aligned}
& \hat{\rho}_{\mathrm{S}}(t)=\operatorname{Tr}_{E}\left(e^{-i \hat{H} t} \hat{\rho}_{0}^{\text {tot }} e^{i \hat{H} t}\right) / \operatorname{Tr}\left(\hat{\rho}_{\mathrm{E}}(t)\right), \\
& \hat{\rho}_{\mathrm{E}}(t)=\operatorname{Tr}_{S}\left(e^{-i \hat{H} t} \hat{\rho}_{0}^{\mathrm{tot}} e^{i \hat{H} t}\right) / \operatorname{Tr}\left(\hat{\rho}_{\mathrm{S}}(t)\right),
\end{aligned}
$$

where $\hat{\rho}_{0}^{\text {tot }}$ is the initial density operator, which we assume as a product state of thermal states in the system and the bath.

$$
\hat{\rho}_{0}^{\text {tot }}=\hat{\rho}_{0} \otimes \hat{\rho}_{E}
$$

Because after a unitary evolution, the trace of $\hat{\rho}_{\mathrm{S}}(t)$ and $\hat{\rho}_{\mathrm{E}}(t)$ is invariant. Therefore we can simply replace $\operatorname{Tr}_{E}\left(\hat{\rho}_{\mathrm{E}}(t)\right)$ to $Z_{\mathrm{E}}=\operatorname{Tr}_{E}\left(\hat{\rho}_{\mathrm{E}}(0)\right)$. Here we employ $\operatorname{Tr}_{E}$ as a trace over the environment's Hilbert space. $\operatorname{Tr}$ is short for $\operatorname{Tr}_{S}$ as trace over the system's Hilbert space.

Following eq. (2.2), the reduced density operator of the system in interaction picture is

$$
\begin{aligned}
\hat{\rho}_{\mathrm{S}}^{\mathrm{I}}(t) & =\operatorname{Tr}_{E}\left(e^{i \hat{H}_{0} t} e^{-i \hat{H} t} \hat{\rho}_{0} \otimes \hat{\rho}_{\mathrm{E}} e^{i \hat{H} t} e^{-i \hat{H}_{0} t}\right) / Z_{\mathrm{E}} \\
& \equiv \operatorname{Tr}_{E}\left(\mathcal{U}(0, t) \hat{\rho}_{0} \otimes \hat{\rho}_{\mathrm{E}} \mathcal{U}(t, 0)\right) / Z_{\mathrm{E}} .
\end{aligned}
$$

The relation between the density matrix operator $\hat{\rho}_{\mathrm{S}}^{\mathrm{I}}(t)$ in interaction picture and the density matrix operator $\hat{\rho}_{\mathrm{S}}(t)$ in Scrödinger's picture is $\hat{\rho}_{\mathrm{S}}(t)=e^{-i \hat{H}_{S} t} \hat{\rho}_{\mathrm{S}}^{\mathrm{I}}(t) e^{i \hat{H}_{S} t}$. Here we introduce the evolution operator $\mathcal{U}(0, t) \equiv e^{i \hat{H}_{0} t} e^{-i \hat{H} t}=\hat{\mathcal{T}}_{t} \exp \left(-i \int_{0}^{t} \hat{V}^{\mathrm{I}}\left(t^{\prime}\right) d t^{\prime}\right)$, where $\hat{V}^{\mathrm{I}}\left(t^{\prime}\right)=e^{i \hat{H}_{0} t} \hat{V} e^{-i \hat{H}_{0} t}$ is in interaction picture, $\hat{\mathcal{T}}_{t}$ is time-ordering operator. By inserting $\hat{\mathcal{U}}(0, t)$ and $\hat{\mathcal{U}}(t, 0)=\hat{\mathcal{U}}(0, t)^{\dagger}$ into eq. (2.5), keeping to $g^{2}$ order, we have

$$
\begin{aligned}
\hat{\rho}_{\mathrm{S}}^{\mathrm{I}}(t)= & \operatorname{Tr}_{E}\left(\left(1-i \int_{0}^{t} \hat{V}^{\mathrm{I}}\left(t^{\prime}\right) d t^{\prime}-\int_{0}^{t} d t_{1} \int_{0}^{t_{1}} d t_{2} \hat{V}^{\mathrm{I}}\left(t_{1}\right) \hat{V}^{\mathrm{I}}\left(t_{2}\right)\right) \hat{\rho}_{0} \otimes \hat{\rho}_{\mathrm{E}}\right. \\
& \left.\left(1+i \int_{0}^{t} \hat{V}^{\mathrm{I}}\left(t^{\prime}\right) d t^{\prime}-\int_{0}^{t} d t_{1} \int_{0}^{t_{1}} d t_{2} \hat{V}^{\mathrm{I}}\left(t_{2}\right) \hat{V}^{\mathrm{I}}\left(t_{1}\right)\right)\right) / Z_{\mathrm{E}} .
\end{aligned}
$$

More explicitly,

$$
\begin{aligned}
\hat{\rho}_{\mathrm{S}}^{\mathrm{I}}(t)=\hat{\rho}_{0} & +g^{2} \int_{0}^{t} d t_{1} \int_{0}^{t_{1}} d t_{2}\left\langle\hat{\xi}_{j}^{\mathrm{I}}\left(t_{1}\right) \hat{\xi}_{\ell}^{\mathrm{I}, \dagger}\left(t_{2}\right)\right\rangle_{\mathrm{E}}\left(\hat{\mathcal{O}}_{\ell}^{\mathrm{I}}\left(t_{2}\right) \hat{\rho}_{0} \hat{\mathcal{O}}_{j}^{\mathrm{I}, \dagger}\left(t_{1}\right)-\hat{\mathcal{O}}_{j}^{\mathrm{I}, \dagger}\left(t_{1}\right) \hat{\mathcal{O}}_{\ell}^{\mathrm{I}}\left(t_{2}\right) \hat{\rho}_{0}\right) \\
& +g^{2} \int_{0}^{t} d t_{1} \int_{0}^{t_{1}} d t_{2}\left\langle\hat{\xi}_{j}^{\mathrm{I}, \dagger}\left(t_{1}\right) \hat{\xi}_{\ell}^{\mathrm{I}}\left(t_{2}\right)\right\rangle_{\mathrm{E}}\left(\hat{\mathcal{O}}_{\ell}^{\mathrm{I}, \dagger}\left(t_{2}\right) \hat{\rho}_{0} \hat{\mathcal{O}}_{j}^{\mathrm{I}}\left(t_{1}\right)-\hat{\mathcal{O}}_{j}^{\mathrm{I}}\left(t_{1}\right) \hat{\mathcal{O}}_{\ell}^{\mathrm{I}, \dagger}\left(t_{2}\right) \hat{\rho}_{0}\right) \\
& +g^{2} \int_{0}^{t} d t_{1} \int_{0}^{t_{1}} d t_{2}\left\langle\hat{\xi}_{j}^{\mathrm{I}}\left(t_{2}\right) \hat{\xi}_{\ell}^{\mathrm{I}, \dagger}\left(t_{1}\right)\right\rangle_{\mathrm{E}}\left(\hat{\mathcal{O}}_{\ell}^{\mathrm{I}}\left(t_{1}\right) \hat{\rho}_{0} \hat{\mathcal{O}}_{j}^{\mathrm{I}, \dagger}\left(t_{2}\right)-\hat{\rho}_{0} \hat{\mathcal{O}}_{j}^{\mathrm{I}, \dagger}\left(t_{2}\right) \hat{\mathcal{O}}_{\ell}^{\mathrm{I}}\left(t_{1}\right)\right) \\
& +g^{2} \int_{0}^{t} d t_{1} \int_{0}^{t_{1}} d t_{2}\left\langle\hat{\xi}_{j}^{\mathrm{I}, \dagger}\left(t_{2}\right) \hat{\xi}_{\ell}^{\mathrm{I}}\left(t_{1}\right)\right\rangle_{\mathrm{E}}\left(\hat{\mathcal{O}}_{\ell}^{\mathrm{I}, \dagger}\left(t_{1}\right) \hat{\rho}_{0} \hat{\mathcal{O}}_{j}^{\mathrm{I}}\left(t_{2}\right)-\hat{\rho}_{0} \hat{\mathcal{O}}_{j}^{\mathrm{I}}\left(t_{2}\right) \hat{\mathcal{O}}_{\ell}^{\mathrm{I}, \dagger}\left(t_{1}\right)\right)
\end{aligned}
$$

where $\langle\cdot\rangle_{\mathrm{E}}=\operatorname{Tr}_{E}\left(\hat{\rho}_{\mathrm{E}} \cdot\right) / Z_{\mathrm{E}}$ is the environment ensemble average. Here we dropped terms like $\left\langle\hat{\xi}^{\mathrm{I}}(t) \hat{\xi}^{\mathrm{I}}\left(t^{\prime}\right)\right\rangle_{\mathrm{E}}$ and $\left\langle\hat{\xi}^{\mathrm{I}, \dagger}(t) \hat{\xi}^{\mathrm{I}, \dagger}\left(t^{\prime}\right)\right\rangle_{\mathrm{E}}$. These terms are anomalous correlation functions of the environment, which are zero when there is no condensation in the environment. Operators with upper index I are in interaction picture. By introducing Green's function for $\hat{\xi}$ field and Lindblad-like operators $\hat{\mathcal{L}}_{j \ell, \mathcal{O}}^{d, e}$, we can simplify the above result as

$$
\hat{\rho}_{\mathrm{S}}^{\mathrm{I}}(t)=\hat{\rho}_{0}+i g^{2} \iint_{0}^{t} d t_{1} d t_{2}\left(G_{\xi, j \ell}^{<}\left(t_{1}, t_{2}\right) \hat{\mathcal{L}}_{j \ell, \mathcal{O}}^{d}\left(t_{1}, t_{2}\right)+G_{\xi, j \ell}^{>}\left(t_{2}, t_{1}\right) \hat{\mathcal{L}}_{j \ell, \mathcal{O}}^{e}\left(t_{1}, t_{2}\right)\right) \hat{\rho}_{0},
$$




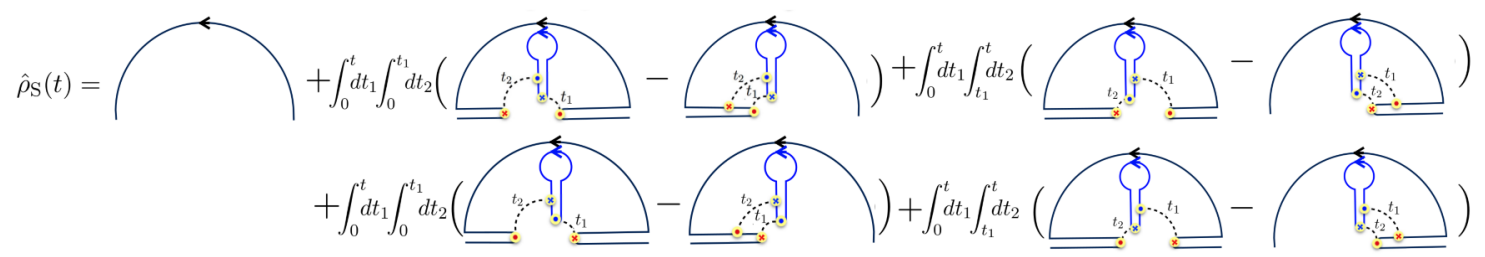

Figure 1. Diagrammatic illustration of eq. (2.7).

where

$$
G_{\xi, j \ell}^{<}\left(t_{1}, t_{2}\right) \equiv-i\left\langle\hat{\xi}_{j}^{\mathrm{I}}\left(t_{1}\right) \hat{\xi}_{\ell}^{\mathrm{I}, \dagger}\left(t_{2}\right)\right\rangle_{\mathrm{E}}, \quad G_{\xi, j \ell}^{>}\left(t_{1}, t_{2}\right) \equiv-i\left\langle\hat{\xi}_{j}^{\mathrm{I}, \dagger}\left(t_{1}\right) \hat{\xi}_{\ell}^{\mathrm{I}}\left(t_{2}\right)\right\rangle_{\mathrm{E}},
$$

$\hat{\mathcal{L}}^{d}$ and $\hat{\mathcal{L}}^{e}$ are dissipation and enhancement operators which are defined as

$$
\begin{aligned}
& \hat{\mathcal{L}}_{j \ell, \mathcal{O}}^{d}\left(t_{1}, t_{2}\right) \hat{\rho}_{0} \equiv \hat{\mathcal{O}}_{\ell}^{\mathrm{I}}\left(t_{2}\right) \hat{\rho}_{0} \hat{\mathcal{O}}_{j}^{\mathrm{I}, \dagger}\left(t_{1}\right)-\theta_{12} \hat{\mathcal{O}}_{j}^{\mathrm{I}, \dagger}\left(t_{1}\right) \hat{\mathcal{O}}_{\ell}^{\mathrm{I}}\left(t_{2}\right) \hat{\rho}_{0}-\theta_{21} \hat{\rho}_{0} \hat{\mathcal{O}}_{j}^{\mathrm{I}, \dagger}\left(t_{1}\right) \hat{\mathcal{O}}_{\ell}^{\mathrm{I}}\left(t_{2}\right), \\
& \hat{\mathcal{L}}_{j \ell, \mathcal{O}}^{e}\left(t_{1}, t_{2}\right) \hat{\rho}_{0} \equiv \hat{\mathcal{O}}_{\ell}^{\mathrm{I}, \dagger}\left(t_{2}\right) \hat{\rho}_{0} \hat{\mathcal{O}}_{j}^{\mathrm{I}}\left(t_{1}\right)-\theta_{12} \hat{\mathcal{O}}_{j}^{\mathrm{I}}\left(t_{1}\right) \hat{\mathcal{O}}_{\ell}^{\mathrm{I}, \dagger}\left(t_{2}\right) \hat{\rho}_{0}-\theta_{21} \hat{\rho}_{0} \hat{\mathcal{O}}_{j}^{\mathrm{I}}\left(t_{1}\right) \hat{\mathcal{O}}_{\ell}^{\mathrm{I}, \dagger}\left(t_{2}\right)
\end{aligned}
$$

Here $\theta_{12}=\theta\left(t_{1}-t_{2}\right)$ is heavyside step function, which is 1 when $t_{1}>t_{2}, 0$ when $t_{1}<t_{2}$, and it is $\frac{1}{2}$ when $t_{1}=t_{2}$. Here we also assumed that $\langle\hat{\xi}(t)\rangle_{\mathrm{E}}=0$. This condition is usually satisfied. Here we assume the vacuum of environment is not a symmetry broken state of $\hat{\xi}_{j}$.

Now we introduce a diagram to describe the above result. Diagrammatically, eq. (2.7) can be expressed as figure 1. The diagram rules are the following, we use red dot as $\hat{\mathcal{O}}^{\dagger}$, red cross as $\hat{\mathcal{O}}$. Here we dropped the subindices of operators $\hat{\mathcal{O}}$. Blue dot is $\hat{\xi}^{\dagger}$ and blue cross is $\hat{\xi}$. The arrow lines are evolution operators whose meanings are explained as below. The half-circle represents an evolution in imaginary time $-e^{-\beta \hat{H}_{\mathrm{S}}}$ and the blue full circle is $e^{-\beta_{\mathrm{E}} \hat{H}_{\mathrm{E}}}$. A closed circle means trace. Every dashed line connecting blue $\operatorname{dot}(\operatorname{cross})$ and red cross(dot) carries a factor $g$. The length of lines in $x$-direction represents real-time, representing $e^{-i \hat{H}_{S} t}$ when the arrow is pointing at the origin of the circle and representing $e^{i \hat{H}_{S} t}$ in the outgoing direction. A closed blue curve represents $G_{\xi}^{>,<}$. One can find, by applying these rules, the diagrams given in figure 1 recover eq. (2.7).

Then we are ready to calculate the Rényi entropy of the system up to $g^{2}$ order. Now we calculate the second Rényi entropy, which is $S_{\mathrm{RE}}^{(2)}=-\log \left(\operatorname{Tr}\left(\hat{\rho}_{\mathrm{S}}^{2}(t)\right)\right)=-\log \left(\operatorname{Tr}\left(\hat{\rho}_{\mathrm{S}}^{\mathrm{I}, 2}(t)\right)\right)$. Here $\operatorname{Tr}$ is a trace over the system's Hilbert space. First we calculate $\operatorname{Tr}\left(\hat{\rho}_{\mathrm{S}}(t)^{2}\right)$,

$$
\begin{aligned}
\operatorname{Tr}\left(\hat{\rho}_{\mathrm{S}}(t)^{2}\right)=\operatorname{Tr}\left(\hat{\rho}_{0}^{2}\right)+2 i g^{2} \sum_{j, \ell} \iint_{0}^{t} d t_{1} d t_{2}\left(G_{\xi, j \ell}^{<}\left(t_{1}, t_{2}\right) \operatorname{Tr}\left(\hat{\rho}_{0}\left(\hat{\mathcal{L}}_{j \ell, \mathcal{O}}^{d}\left(t_{1}, t_{2}\right) \hat{\rho}_{0}\right)\right)\right. \\
\left.+G_{\xi, j \ell}^{>}\left(t_{2}, t_{1}\right) \operatorname{Tr}\left(\hat{\rho}_{0}\left(\hat{\mathcal{L}}_{j \ell, \mathcal{O}}^{e}\left(t_{1}, t_{2}\right) \hat{\rho}_{0}\right)\right)\right) .
\end{aligned}
$$

The above expression can also be displayed in diagrammatic language as is shown in figure 2 .

Now we discuss two situations, the first case is $\hat{\mathcal{O}}_{j}$ and $\hat{\xi}_{j}$ being both bosonic annihilation operators. Both in Green's function and the $\hat{\mathcal{L}}$ operators, there are sub-indices $j$ and $\ell$. Here we assume $G_{\xi, j \ell}^{>,<}=G_{\xi, j}^{>,<} \delta_{j \ell}$. Then only $\hat{\mathcal{L}}_{j j, \mathcal{O}}^{d, e}$ contributes. In this diagonal basis, we assume that $G_{\xi, j}^{>,<}$is $j$ independent, then we can denote $G_{\xi, j}^{>,<}$as $G_{\xi}^{>,<}$. According to the 


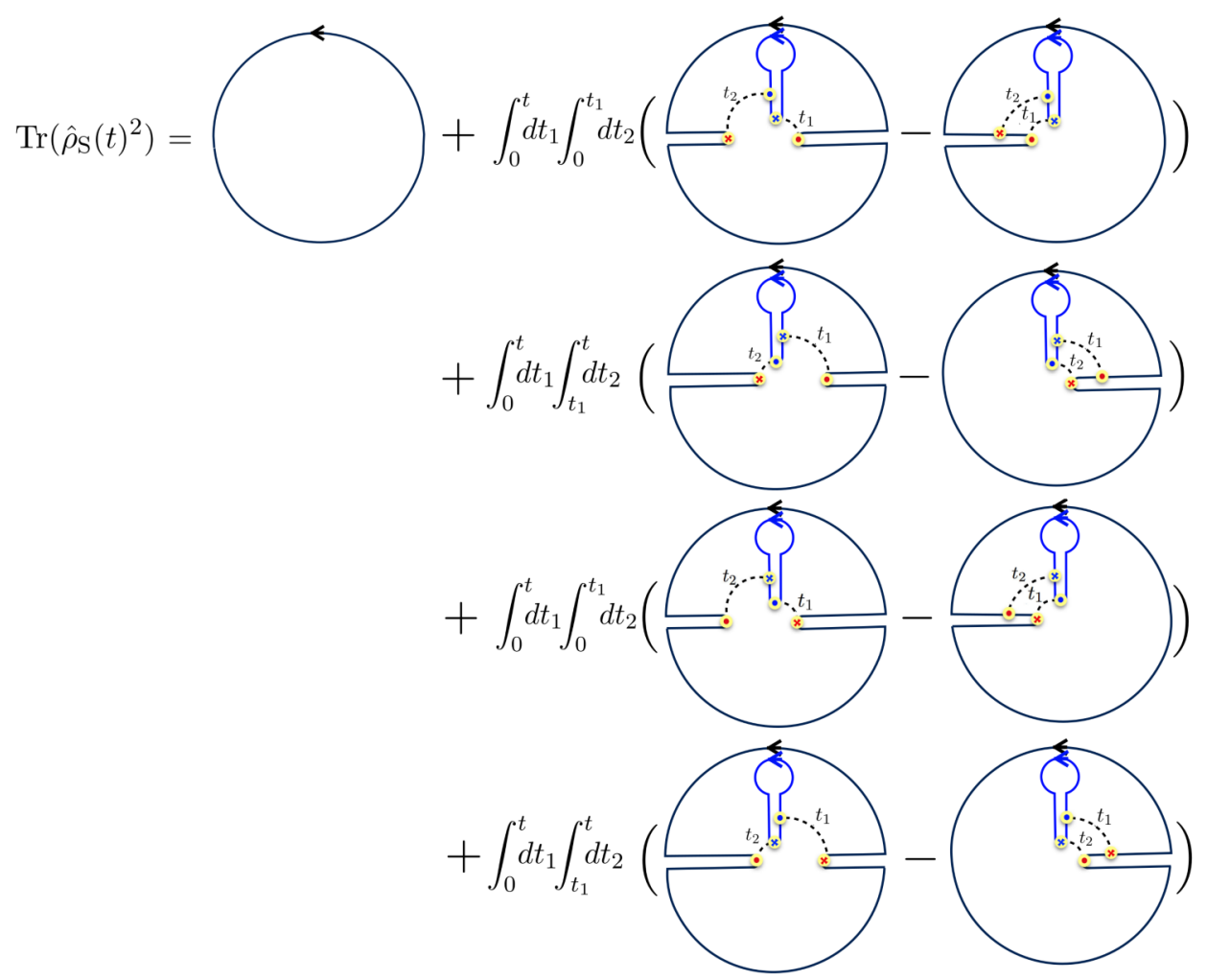

Figure 2. Perturbation digrams for $\operatorname{Tr}\left(\hat{\rho}_{\mathrm{S}}^{2}(t)\right)$, up to $g^{2}$ order. Diagram representation of eq. (2.11).

relation between the greater (lesser) Green's function and the spectral function, we have

$$
\begin{aligned}
& G_{\xi}^{>}\left(t_{1}, t_{2}\right)=-i \int d \omega^{\prime} \mathcal{A}_{\xi}\left(\omega^{\prime}, \beta_{\mathrm{E}}\right) \frac{1}{e^{\beta_{E} \omega^{\prime}}-1} e^{i \omega^{\prime}\left(t_{1}-t_{2}\right)}, \\
& G_{\xi}^{<}\left(t_{1}, t_{2}\right)=-i \int d \omega^{\prime} \mathcal{A}_{\xi}\left(\omega^{\prime}, \beta_{\mathrm{E}}\right) \frac{e^{\beta_{E} \omega^{\prime}}}{e^{\beta_{E} \omega^{\prime}}-1} e^{-i \omega^{\prime}\left(t_{1}-t_{2}\right)},
\end{aligned}
$$

where $\mathcal{A}_{\xi}\left(\omega, \beta_{\mathrm{E}}\right)$ is the spectral function of $\hat{\xi}$ field, $\mathcal{A}_{\xi}\left(\omega, \beta_{\mathrm{E}}\right)=-\frac{1}{\pi} \operatorname{Im} G_{\xi}^{R}(\omega)$ where $G_{\xi}^{R}(t)=-i \theta(t)\left\langle\left[\hat{\xi}(t), \xi^{\dagger}(0)\right]\right\rangle_{\mathrm{E}}$ is the retard Green's function of $\hat{\xi}$ field. $1 / \beta_{E}$ is the environment temperature. Here we can see, a white noise bath can be generated in two cases. At high temperature, $\beta_{E}$ is close to zero, therefore when $\mathcal{A}_{\xi}\left(\omega, \beta_{\mathrm{E}}\right) \sim \omega_{\mathrm{E}}$, both $G_{\xi}^{>}\left(t_{1}, t_{2}\right)$ and $G_{\xi}^{<}\left(t_{1}, t_{2}\right) \propto \delta\left(t_{1}-t_{2}\right)$. This is an Ohm bath at a high temperature. At low temperature, we find $G_{\xi}^{>} \sim 0$, and white noise is generated by a constant density of states $\mathcal{A}_{\xi}\left(\omega, \beta_{\mathrm{E}}\right)$, which is independent of frequency. In these two cases, we can reach Markovian limit.

On the other hand, we find

$$
\operatorname{Tr}\left(\hat{\rho}_{0} \hat{\mathcal{L}}_{j j, \mathcal{O}}^{d}\left(t_{1}, t_{2}\right) \hat{\rho}_{0}\right)=\operatorname{Tr}\left(\hat{\rho}_{0} \hat{\mathcal{O}}_{j}^{\mathrm{I}, \dagger}\left(t_{1}\right) \hat{\rho}_{0} \hat{\mathcal{O}}_{j}^{\mathrm{I}}\left(t_{2}\right)\right)-\operatorname{Tr}\left(\hat{\rho}_{0}^{2} \hat{\mathcal{O}}_{j}^{\mathrm{I}, \dagger}\left(t_{1}\right) \hat{\mathcal{O}}_{j}^{\mathrm{I}}\left(t_{2}\right)\right),
$$

and

$$
\operatorname{Tr}\left(\hat{\rho}_{0} \hat{\mathcal{L}}_{j j, \mathcal{O}}^{e}\left(t_{2}, t_{1}\right) \hat{\rho}_{0}\right)=\operatorname{Tr}\left(\hat{\rho}_{0} \hat{\mathcal{O}}_{j}^{\mathrm{I}, \dagger}\left(t_{1}\right) \hat{\rho}_{0} \hat{\mathcal{O}}_{j}^{\mathrm{I}}\left(t_{2}\right)\right)-\operatorname{Tr}\left(\hat{\rho}_{0}^{2} \hat{\mathcal{O}}_{j}^{\mathrm{I}}\left(t_{2}\right) \hat{\mathcal{O}}_{j}^{\mathrm{I}, \dagger}\left(t_{1}\right)\right)
$$


If we define the average spectrum function $\mathcal{A}_{\mathcal{O}}(\omega, 2 \beta)$ as

$$
\mathcal{A}_{\mathcal{O}}(\omega, 2 \beta)=-\frac{1}{\pi N_{j}} \sum_{j} \operatorname{Im} G_{j, \mathcal{O}}^{R}(\omega, 2 \beta),
$$

where $G_{j, O}^{R}(\omega, 2 \beta)$ is the Fourier transformation of retard Green's function $G_{j, \mathcal{O}}^{R}(t)$ and $G_{j, \mathcal{O}}^{R}(t)=-i \theta(t) \operatorname{Tr}\left(\hat{\rho}_{0}^{2}\left[\mathcal{O}_{j}^{\mathrm{I}}(t), \mathcal{O}^{\mathrm{I}, \dagger}(0)\right]\right) / \operatorname{Tr}\left(\hat{\rho}_{0}^{2}\right) . N_{j}$ is the number of $\mathrm{j}$ index. Applying the Lehmann spectrum representation theorem, we have

$$
\sum_{j} \operatorname{Tr}\left(\hat{\rho}_{0} \hat{\mathcal{O}}_{j}^{\mathrm{I}, \dagger}\left(t_{1}\right) \hat{\rho}_{0} \hat{\mathcal{O}}_{j}^{\mathrm{I}}\left(t_{2}\right)\right)=N_{j} \operatorname{Tr}\left(\hat{\rho}_{0}^{2}\right) \int d \omega \mathcal{A}_{\mathcal{O}}(\omega, 2 \beta) \frac{e^{\beta \omega}}{e^{2 \beta \omega}-1} e^{i \omega\left(t_{1}-t_{2}\right)},
$$

and

$$
\begin{aligned}
& \sum_{j} \operatorname{Tr}\left(\hat{\rho}_{0}^{2} \hat{\mathcal{O}}_{j}^{\mathrm{I}, \dagger}\left(t_{1}\right) \hat{\mathcal{O}}_{j}^{\mathrm{I}}\left(t_{2}\right)\right)=N_{j} \operatorname{Tr}\left(\hat{\rho}_{0}^{2}\right) \int d \omega \mathcal{A}_{\mathcal{O}}(\omega, 2 \beta) \frac{1}{e^{2 \beta \omega}-1} e^{i \omega\left(t_{1}-t_{2}\right)} \\
& \sum_{j} \operatorname{Tr}\left(\hat{\rho}_{0}^{2} \hat{\mathcal{O}}_{j}^{\mathrm{I}}\left(t_{1}\right) \hat{\mathcal{O}}_{j}^{\mathrm{I}, \dagger}\left(t_{2}\right)\right)=N_{j} \operatorname{Tr}\left(\hat{\rho}_{0}^{2}\right) \int d \omega \mathcal{A}_{\mathcal{O}}(\omega, 2 \beta) \frac{e^{2 \beta \omega}}{e^{2 \beta \omega}-1} e^{-i \omega\left(t_{1}-t_{2}\right)} .
\end{aligned}
$$

By inserting eq. (2.12), eq. (2.13), eq. (2.17), eq. (2.18) and eq. (2.19) into eq. (2.11), the deviation of Renyi entropy $\delta S_{\mathrm{RE}}^{(2)}(t) \equiv S_{\mathrm{RE}}^{(2)}(t)+\log \left(\operatorname{Tr}\left(\hat{\rho}_{0}^{2}\right)\right)$ is

$$
\delta S_{\mathrm{RE}}^{(2)}(t)=4 g^{2} N_{j} \int_{0}^{t} d t_{0} \int d \omega \int d \omega^{\prime} \frac{\sin \left(\omega-\omega^{\prime}\right) t_{0}}{\omega-\omega^{\prime}} \mathcal{W}_{\beta \beta_{E}}^{B}\left(\omega, \omega^{\prime}\right) \mathcal{A}_{\xi}\left(\omega^{\prime}, \beta_{\mathrm{E}}\right) \mathcal{A}_{\mathcal{O}}(\omega, 2 \beta),
$$

where

$$
\mathcal{W}_{\beta \beta_{E}}^{B}\left(\omega, \omega^{\prime}\right)=\frac{\left(e^{\beta \omega}-1\right)\left(e^{\beta \omega}-e^{\beta_{E} \omega^{\prime}}\right)}{\left(e^{2 \beta \omega}-1\right)\left(e^{\beta_{E} \omega^{\prime}}-1\right)} .
$$

According to this formula, we learn, the entropy response is only dependent on the distribution function and the spectrum of the bath and the system.

As the entropy response theory at $g^{2} N_{j}$ order is indeed an exponential contribution in $\operatorname{Tr}\left(\hat{\rho}_{S}^{2}(t)\right)$ 's perturbation, therefore the response theory for entropy may have a larger applicable region. We can apply a perturbation calculation to the next order $g^{4}$, where we can capture how the spectrum function of the system and the environment is changed by interactions. But these back action effects are not considered in the present calculation.

Quite similarly, we can work out the Rényi entropy response for a fermionic bath and fermionic operators $\hat{\mathcal{O}}_{j}$, which is

$$
\delta S_{\mathrm{RE}}^{(2)}(t)=4 g^{2} N_{j} \int_{0}^{t} d t_{0} \int d \omega \int d \omega^{\prime} \frac{\sin \left(\omega-\omega^{\prime}\right) t_{0}}{\omega-\omega^{\prime}} \mathcal{W}_{\beta \beta_{E}}^{F}\left(\omega, \omega^{\prime}\right) \mathcal{A}_{\xi}\left(\omega^{\prime}, \beta_{\mathrm{E}}\right) \mathcal{A}_{\mathcal{O}}(\omega, 2 \beta),
$$

where

$$
\mathcal{W}_{\beta \beta_{E}}^{F}\left(\omega, \omega^{\prime}\right)=\frac{\left(e^{\beta \omega}-1\right)\left(e^{\beta \omega}-e^{\beta_{E} \omega^{\prime}}\right)}{\left(1+e^{2 \beta \omega}\right)\left(1+e^{\beta_{E} \omega^{\prime}}\right)} .
$$

Notice that in the fermionic case, the Markovian condition is requiring a frequency independent spectrum together with a frequency independent distribution function. The latter condition is satisfied only for zero and infinite temperature. Therefore there are two ways to break down Markovian approximation, one is by finite temperature; another is by adding strong frequency dependence in the noise spectrum of the environment. 


\subsection{General features for bosonic systems}

In this section, we first discuss the short time and the long time behavior of the Rényi entropy response for a bosonic environment. Then we derive a "Fourier law " in heat transport when the system and the environment have a small temperature difference.

First, we discuss the short time and long time dynamics of $\delta S_{\mathrm{RE}}^{(2)}$. When $t$ is small, in a sense $\mathcal{J} t \ll 1, \mathcal{J}^{\prime} t \ll 1\left(\mathcal{J}\right.$ and $\mathcal{J}^{\prime}$ are typical energy scale for the system and the bath respectively), we have

$$
\frac{\sin \left(\omega-\omega^{\prime}\right) t_{0}}{\omega-\omega^{\prime}} \approx t_{0}
$$

For this reason, the short time dynamics is always proportional to $t^{2}$.

$$
\delta S_{\mathrm{RE}}^{(2)}(t)=\frac{1}{2} \kappa_{\mathrm{B}} t^{2}
$$

where the coefficient is

$$
\kappa_{\mathrm{B}} \equiv 4 g^{2} N_{j} \int d \omega \int d \omega^{\prime} \mathcal{W}_{\beta \beta_{E}}^{B}\left(\omega, \omega^{\prime}\right) \mathcal{A}_{\mathcal{O}}(\omega, 2 \beta) \mathcal{A}_{\xi}\left(\omega^{\prime}, \beta_{\mathrm{E}}\right) .
$$

The bosonic environment is unique because the chemical potential of Bose distribution is negative (although in our formula we take chemical potential to be zero), and the frequency of bosonic excitations is always positive. Therefore when $\beta_{\mathrm{E}}>\beta$ and the environment has a lower temperature, $\kappa_{\mathrm{B}}$ is usually negative. Further

$$
\mathcal{W}_{\beta \beta_{E}}^{B}\left(\omega, \omega^{\prime}\right)=\frac{e^{\beta \omega}-e^{\beta_{E} \omega^{\prime}}}{\left(e^{\beta \omega}+1\right)\left(e^{\beta_{\mathrm{E}} \omega^{\prime}}-1\right)} \approx \frac{e^{\beta \omega-\beta_{\mathrm{E}} \omega^{\prime}}-1}{e^{\beta \omega}+1} .
$$

As $\omega$ and $\omega^{\prime}$ are both positive, as long as $\beta \omega<\beta_{\mathrm{E}} \omega^{\prime}$, the factor $\mathcal{W}_{\beta \beta_{E}}^{B}\left(\omega, \omega^{\prime}\right)$ is negative. Of course, this is only part of the integration, which can not determine the total sign of $\kappa_{\mathrm{B}}$. Only when the integration over the region where $\beta \omega<\beta_{\mathrm{E}} \omega^{\prime}$ is dominant over other regions, $\kappa_{\mathrm{B}}$ is then negative. So it is quite possible that $\kappa_{\mathrm{B}}<0$. Later we will see it is different in the fermionic bath case.

Now we turn to long time case. Here we assume $t$ is large so that $\mathcal{J} t_{0} \sim \mathcal{J}^{\prime} t_{0} \sim 1$, we have

$$
\frac{\sin \left(\omega-\omega^{\prime}\right) t_{0}}{\omega-\omega^{\prime}} \approx \pi \delta\left(\omega-\omega^{\prime}\right)
$$

Therefore the long time dynamics is approximately

$$
\delta S_{\mathrm{RE}}^{(2)}(t)=4 \pi g^{2} N_{j} t \int d \omega \mathcal{W}_{\beta \beta_{E}}^{B}(\omega, \omega) \mathcal{A}_{\xi}\left(\omega, \beta_{\mathrm{E}}\right) \mathcal{A}_{\mathcal{O}}(\omega, 2 \beta) .
$$

The sign for the entropy growth rate in a long time limit is determined by the sign of $\mathcal{W}_{\beta \beta_{E}}^{B}(\omega, \omega) . \mathcal{W}_{\beta \beta_{E}}^{B}(\omega, \omega)>0$ when $\beta_{\mathrm{E}}<\beta$. In this situation, the environment's temperature is higher than the system's. $\mathcal{W}_{\beta \beta_{E}}^{B}(\omega, \omega)<0$ when $\beta_{\mathrm{E}}>\beta$. In this situation, the environment has a lower temperature than the system.

Now we are going to derive a Fourier heat transport formula from our eq. (2.26). Now we assume two temperatures $T=1 / \beta$ and $T_{\mathrm{E}}=1 / \beta_{\mathrm{E}}$ are close. Here we have taken $k_{\mathrm{B}}=1$. Further, we assume $\beta_{\mathrm{E}}$ is small, then we have

$$
\mathcal{W}_{\beta \beta_{E}}^{B}(\omega, \omega)=\frac{1}{e^{\beta \omega}+1} \frac{e^{\left(\beta-\beta_{\mathrm{E}}\right) \omega}-1}{1-e^{-\beta_{\mathrm{E}} \omega}} \approx \frac{T_{\mathrm{E}}-T}{T} \frac{1}{\left(e^{\beta \omega}+1\right)} .
$$


Insert eq. (2.27) into eq. (2.26), we have

$$
T \delta S_{\mathrm{RE}}^{(2)}(t)=4 \pi g^{2} N_{j}\left(\int_{0}^{\infty} d \omega \frac{\mathcal{A}_{\mathcal{O}}(\omega, 2 \beta) \mathcal{A}_{\xi}\left(\omega, 2 \beta_{\mathrm{E}}\right)}{e^{\beta \omega}+1}\right)\left(T_{\mathrm{E}}-T\right) t .
$$

If we can define $d Q \equiv T \delta S_{\mathrm{RE}}^{(2)}(t)$ as some kind of heat, therefore above equation shows a Fourier law of heat transport. The heat transport rate is proportional to the temperature difference between the system and the bath. If we define $F_{\mathrm{Q}}=d Q / d t /\left(T_{\mathrm{E}}-T\right)$, then the transport coefficient $F_{\mathrm{Q}}$ is predicted as

$$
F_{Q}=4 \pi g^{2} N_{j} \int_{0}^{\infty} d \omega \frac{\mathcal{A}_{\mathcal{O}}(\omega, 2 \beta) \mathcal{A}_{\xi}\left(\omega, \beta_{\mathrm{E}}\right)}{e^{\beta \omega}+1} .
$$

\subsection{General features for fermionic systems}

In this section, we discuss the short time and long time entropy growth for the fermionic bath. Meanwhile, we will give the " heat transport " coefficient for the fermionic bath.

First, we discuss the short-time behavior of $\delta S_{\mathrm{RE}}^{(2)}(t)$. Similar to the case of the bosonic system, we have

$$
\delta S_{\mathrm{RE}}^{(2)}(t)=\frac{1}{2} \kappa_{\mathrm{F}} t^{2}
$$

where

$$
\kappa_{\mathrm{F}}=4 g^{2} N_{j} \int d \omega \int d \omega^{\prime} \mathcal{W}_{\beta \beta_{E}}^{F}\left(\omega, \omega^{\prime}\right) \mathcal{A}_{\mathcal{O}}(\omega, 2 \beta) \mathcal{A}_{\xi}\left(\omega^{\prime}, \beta_{\mathrm{E}}\right) .
$$

The only difference compared with eq. (2.25) is that the distribution function $\mathcal{W}_{\beta \beta_{E}}^{B}$ is replaced by $\mathcal{W}_{\beta \beta_{E}}^{F}$. For fermions, negative frequency is meaningful because of the presence of Fermi level. Here Fermi level is taken to be zero. At low temperature,

$$
\mathcal{W}_{\beta \beta_{E}}^{F}\left(\omega, \omega^{\prime}\right) \approx \frac{\left(e^{\beta \omega}-1\right)\left(e^{\beta \omega-\beta_{E} \omega^{\prime}}-1\right)}{e^{2 \beta \omega}+1}
$$

It is clear that when $\omega<0, \omega^{\prime}>0, \mathcal{W}_{\beta \beta_{E}}^{F}\left(\omega, \omega^{\prime}\right)>0$. One can find when the system temperature is low, the integration is dominant by the occupied frequency $\omega<0$. Therefore $\kappa_{\mathrm{F}}$ is more likely to be positive rather than being negative. This is very different from the bosonic case. Of course, it is an argument rather than a proof of $\kappa_{F / B}$ 's sign.

Now we consider the long-time behavior of $\delta S_{\mathrm{RE}}^{(2)}(t)$. In the long-time limit, we have

$$
\delta S_{\mathrm{RE}}^{(2)}(t)=4 \pi g^{2} N_{j} t \int d \omega \mathcal{W}_{\beta \beta_{E}}^{F}(\omega, \omega) \mathcal{A}_{\xi}\left(\omega, \beta_{\mathrm{E}}\right) \mathcal{A}_{\mathcal{O}}(\omega, 2 \beta) .
$$

One can prove that for $\beta_{\mathrm{E}}>\beta, \mathcal{W}_{\beta \beta_{E}}^{F}(\omega, \omega)<0$ for any $\omega$. Therefore if the environment has a larger initial temperature, $\delta S_{\mathrm{RE}}^{(2)}(t)$ experienced a linear growth and if the environmental temperature is lower than the system's, $\delta S_{\mathrm{RE}}^{(2)}(t)$ is decreasing linearly in the long-time limit. However, as we stated previously, the short-time dynamics of the low-temperature fermionic bath is an entropy growth, therefore the entropy dynamics could be non-monotonic. Later in the next section, we will show this case in a specific example. Here we stress that the kink in $\delta S_{\mathrm{RE}}^{(2)}(t)$ could be very general in fermionic case, not necessarily as an interpretation of gravity duality. 
Finally, we discuss the situation when the system and the bath's temperature difference is small. Again a Fourier-alike law can be presented,

$$
\mathcal{W}_{\beta \beta_{E}}^{F}(\omega, \omega)=\frac{e^{\beta \omega}-1}{e^{2 \beta \omega}+1} \frac{e^{\left(\beta-\beta_{\mathrm{E}}\right) \omega}-1}{1+e^{-\beta_{\mathrm{E}} \omega}} \approx \frac{\left(e^{\beta \omega}-1\right) \omega \beta \beta_{\mathrm{E}}}{\left(e^{2 \beta \omega}+1\right)\left(1+e^{-\beta_{\mathrm{E}} \omega}\right)}\left(T_{\mathrm{E}}-T\right)
$$

Again we can get a " Fourier law " in " heat transport " if we define $T \delta S_{\mathrm{RE}}^{(2)}(t)$ as " heat flow " $-d Q$. Again we define $F_{\mathrm{Q}}=d Q / d t /\left(T_{\mathrm{E}}-T\right)$, and we find

$$
F_{\mathrm{Q}}=4 \pi g^{2} N_{j} \int_{-\infty}^{\infty} d \omega \frac{\mathcal{A}_{\mathcal{O}}(\omega, 2 \beta) \mathcal{A}_{\xi}\left(\omega, \beta_{\mathrm{E}}\right)\left(e^{\beta \omega}-1\right) \omega \beta_{\mathrm{E}}}{\left(e^{2 \beta \omega}+1\right)\left(1+e^{-\beta_{\mathrm{E}} \omega}\right)}
$$

\section{Renyi entropy response in SYK model}

In this section, we apply the general Rényi entropy response theory to Sachdev-Ye-Kitaev (SYK) model with a non-Markovian environment. We assume the system is $\mathrm{SYK}_{4}$ whose Hamiltonian is,

$$
\hat{H}_{\mathrm{S}}=\sum_{j k l m} J_{j k l m} \hat{\chi}_{j} \hat{\chi}_{k} \hat{\chi}_{l} \hat{\chi}_{m}
$$

where $\hat{\chi}_{j}$ is Majorana fermion operator. $J_{j k l m}$ are random, and we have

$$
\overline{J_{j k l m}}=0, \quad \overline{J_{j k l m}^{2}}=\frac{3 !}{N^{3}} \mathcal{J}^{2},
$$

where $\#$ is disorder average, $N$ is the total modes number. Throughout our discussion, we are in the conformal limit of the SYK model, so that $N \gg \beta \mathcal{J} \gg 1$.

Here we are going to consider two kinds of baths. One bath is a $\mathrm{SYK}_{2}$ model with zero chemical potential. In this model, when the coupling strength is large, then the spectral function around zero frequency is a constant. This kind of bath has a Markovian limit. Another bath is two $\mathrm{SYK}_{2}$ models with opposite on-site energies, which is more like a gapped system and is far away from a Markovian environment. Here we stress that the non-Markovian effect comes from both the frequency dependence in the spectral function of the environment as well as the distribution function kernel $\mathcal{W}^{F}$. Therefore we separate these two factors with a constant spectral function and a strongly frequency-dependent spectral function. In the former, the non-Markovian effect comes from the distribution function, while in the latter, the non-Markovian effect comes from the spectral function.

\subsection{Heating and evaporation with Markovian-like environment}

Here we assume the environment is random free fermions, $\mathrm{SYK}_{2}$, whose Hamiltonian is

$$
\hat{H}_{\mathrm{E}}=\sum_{\alpha=1}^{M} \sum_{j k=1}^{N} J_{j k}^{\prime} \hat{\psi}_{j, \alpha}^{\dagger} \hat{\psi}_{k, \alpha},
$$

where $\hat{\psi}_{j}$ are complex fermion annihilation operators. $J_{j k}^{\prime}$ is random, and it satisfies

$$
\overline{J_{j k}^{\prime}}=0, \quad \overline{\left(J_{j k}^{\prime}\right)^{2}}=\frac{1}{4 N}\left(\mathcal{J}^{\prime}\right)^{2}
$$


The interaction between the system and the bath is $\hat{V}$,

$$
\hat{V}=g \sum_{j=1}^{N} \sum_{\alpha=1}^{M}\left(\hat{\chi}_{j} \hat{\psi}_{j, \alpha}^{\dagger}+\hat{\psi}_{j, \alpha} \hat{\chi}_{j}\right)
$$

One advantage is that the SYK model is known to have a gravitational duality. SYK model's low energy effective action can be described by a Schwarzian theory, which is dual to the Jackiw-Teitelboim (JT) gravity theory in $\mathrm{AdS}_{2}$ spacetime [23, 24]. The present situation of sudden coupling between the $\mathrm{SYK}_{4}$ and $\mathrm{SYK}_{2}$ model can be viewed as an black hole evaporation problem if the environment's temperature is much lower than the black hole. On the gravitational side, it is known that evaporation experience an entanglement entropy growth and drop, which is well-known as the Page curve. The entanglement entropy calculation based on the RT formula is done recently [39-41] towards an understanding of the Page curve. Therefore, according to the holographic principle, we expect similar nonmonotonic behavior in $\mathrm{SYK}_{4}$ 's entanglement entropy dynamics.

Now we apply the perturbation theory to calculate the Rényi entropy dynamics after a sudden coupling between $\mathrm{SYK}_{4}$ and $\mathrm{SYK}_{2}$ by $\hat{V}$ to see how the second Rényi entropy response to the sudden coupling between these two systems.

From our formula, we find the most important information for Rényi entropy response is the spectrum of the system and the spectrum of the environment. These spectra can be obtained by calculating retard Green's function. Compared with the general theory, we find $\hat{\mathcal{O}}_{j}=\hat{\chi}_{j}$ and $\hat{\xi}_{j}=\hat{\psi}_{j, \alpha}$. Then the spectral function for $\hat{\mathcal{O}}_{j}$ field is

$$
\mathcal{A}_{\mathcal{O}}(\omega, \beta)=\frac{b}{\sqrt{\beta}} \operatorname{Re}\left(\frac{\Gamma\left(\frac{1}{4}-i \beta \omega / 2 \pi\right)}{\Gamma\left(\frac{3}{4}-i \beta \omega / 2 \pi\right)}\right),
$$

where $b=\left(1 / 4 \pi \mathcal{J}^{2}\right)^{1 / 4}$ and $\operatorname{Re}(\#)$ gets the real part of \#. The spectral function of the environment is

$$
\mathcal{A}_{\xi}\left(\omega, \beta_{\mathrm{E}}\right)=\sqrt{1-\frac{\omega^{2}}{\mathcal{J}^{\prime 2}}}
$$

According to the entropy response theory

$$
\delta S_{\mathrm{RE}}^{(2)}(t)=4 g^{2} N M \int_{0}^{t} d t_{0} \int_{-\mathcal{J}^{\prime}}^{\mathcal{J}^{\prime}} d \omega^{\prime} \int_{-\mathcal{J}}^{\mathcal{J}} d \omega \frac{\sin \left(\omega-\omega^{\prime}\right) t_{0}}{\omega-\omega^{\prime}} \mathcal{W}_{\beta \beta_{E}}^{F}\left(\omega, \omega^{\prime}\right) \mathcal{A}_{\mathcal{O}}(\omega, 2 \beta) \mathcal{A}_{\xi}\left(\omega^{\prime}, \beta_{\mathrm{E}}\right) .
$$

In short-time limit, $\sin \left(\omega-\omega^{\prime}\right) t_{0} \sim\left(\omega-\omega^{\prime}\right) t_{0}$, therefore we have

$$
\delta S_{\mathrm{RE}}^{(2)}(t)=\frac{1}{2} \kappa_{F} t^{2}
$$

where $\kappa_{F}=4 g^{2} N M / \operatorname{Tr}\left(\hat{\rho}_{0}^{2}\right) \int_{-\mathcal{J}^{\prime}}^{\mathcal{J}^{\prime}} d \omega^{\prime} \int_{-\mathcal{J}}^{\mathcal{J}} d \omega \mathcal{W}_{\beta \beta_{E}}^{F}\left(\omega, \omega^{\prime}\right) \mathcal{A}_{\mathcal{O}}(\omega, 2 \beta) \mathcal{A}_{\xi}\left(\omega^{\prime}, \beta_{\mathrm{E}}\right)$. In long-time limit, $\sin \left(\omega-\omega^{\prime}\right) t_{0} /\left(\omega-\omega^{\prime}\right) \approx \pi \delta\left(\omega-\omega^{\prime}\right)$, therefore

$$
\delta S_{\mathrm{RE}}^{(2)}(t)=\lambda t,
$$

where $\lambda$ is the entropy rate, whose value is

$$
\lambda=4 \pi g^{2} N M \int_{-\min \left(\mathcal{J}^{\prime}, \mathcal{J}\right)}^{\min \left(\mathcal{J}^{\prime}, \mathcal{J}\right)} d \omega \mathcal{W}_{\beta \beta_{E}}^{F}(\omega, \omega) \mathcal{A}_{\mathcal{O}}(\omega, 2 \beta) \mathcal{A}_{\xi}\left(\omega, \beta_{\mathrm{E}}\right)
$$




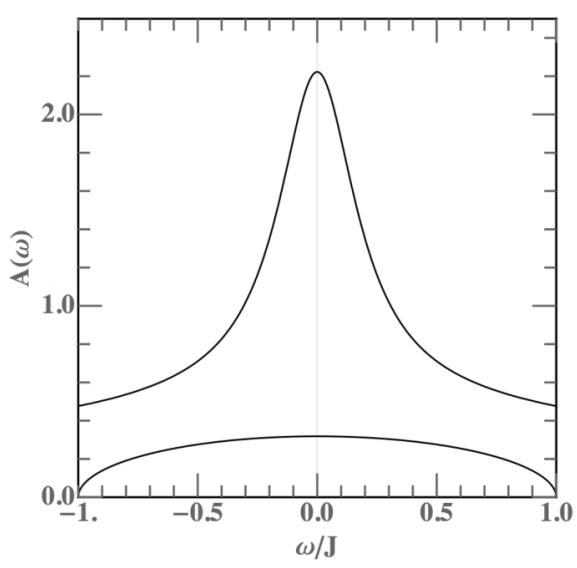

(a)

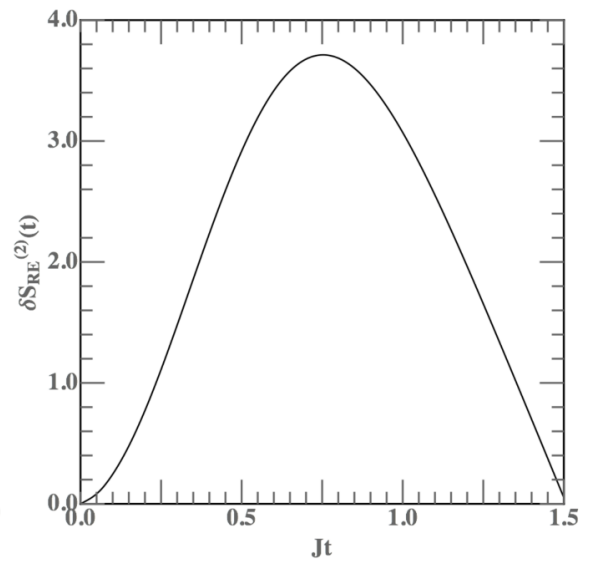

(b)

Figure 3. Evaporation of "Black Hole", In (a) we show the spectral function of the system $\left(\mathrm{SYK}_{4}\right.$ model, $\beta=2, \mathcal{J}=2)$ and the environment $\left(\mathrm{SYK}_{2}\right.$ model with $\left.\beta=20, \mathcal{J}^{\prime}=2\right)$; in (b), we show the Rényi entropy response of the sudden coupling between SYK model and the environment. As the environment is at low temperature, it is an evaporation process. We can see a linear growth follows a quadratic growth, then a sharp linear drop follows. The shape of the curve is alike the Page curve in black hole evaporation. $g^{2} N M$ is tuned for showing the figures, but these values are not important at this level.

Here $\min (a, b)$ takes the smaller one in $a$ and $b$. Here $\lambda$ can be negative. The general picture for the entanglement entropy growth is a quadratic growth at an early time and then crossover to linear growth (or decrease) at a late time. As the late time behavior can be a decrease of entropy, therefore we can see the signal of evaporation in our perturbative calculation even if it is not early time behavior.

Here we stress that for $\sin \left(\omega-\omega^{\prime}\right) t_{0} /\left(\omega-\omega^{\prime}\right) \propto \delta\left(\omega-\omega^{\prime}\right)$, we need a really large $t_{0}$. Indeed this approximation is better satisfied when $\min \left(\mathcal{J}^{\prime}, \mathcal{J}\right) t_{0}$ is really large. Therefore before that time scale, there are always oscillations in $\delta S_{\mathrm{RE}}^{(2)}(t)$, but these oscillations are slow. In the long-time limit, we find only if $\beta=\beta_{\mathrm{E}}$, we have $\delta S_{\mathrm{RE}}^{(2)}=0$. However, in some periods of time, $\delta S_{\mathrm{RE}}^{(2)} \sim 0$ for quite a remarkable time scale, which may seems like prethermalization. In this section, we only focus on the situation where $\beta$ and $\beta_{\mathrm{E}}$ have a large difference.

In the following, we present the numerical calculation for mainly two kinds of processes. First of all, we discuss the situation of heating where the system is at low temperature and the environment is at high temperature. As is shown in figure 4 , we couple an $\mathrm{SYK}_{4}$ model with $\mathcal{J}=4, \beta=10$ to an $\mathrm{SYK}_{2}$ model with $\mathcal{J}^{\prime}=8$ and $\beta_{\mathrm{E}}=2$. This situation mimics a Black Hole coupling to a hot environment. Just as our analysis in the last section, the Rényi entropy experienced a quadratic growth in the short-time limit and then crossover to linear growth on long-time scale.

Second, we study an $\mathrm{SYK}_{4}$ model with $\mathcal{J}=2, \beta=2$ coupling to an $\mathrm{SYK}_{2}$ model with $\mathcal{J}^{\prime}=2$, and $\beta_{\mathrm{E}}=20$. The environment is at low temperature, hence it describes a "Black Hole " evaporation process. A Page curve alike behavior is expected and we find that even if the method is perturbative, the non-monotonic behavior can be captured as is shown in figure 3 . 


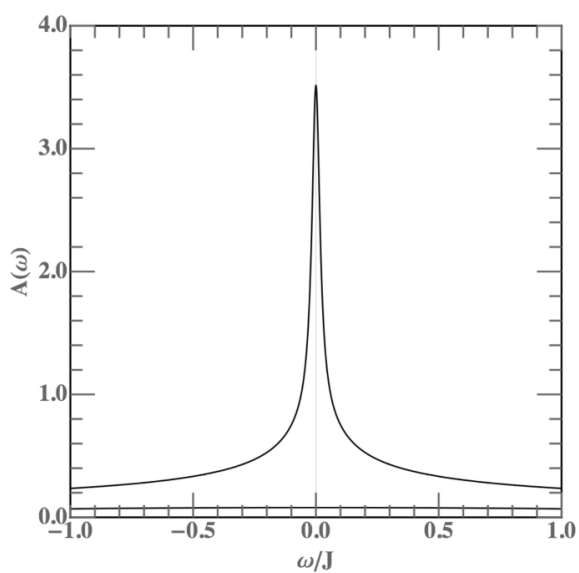

(a)

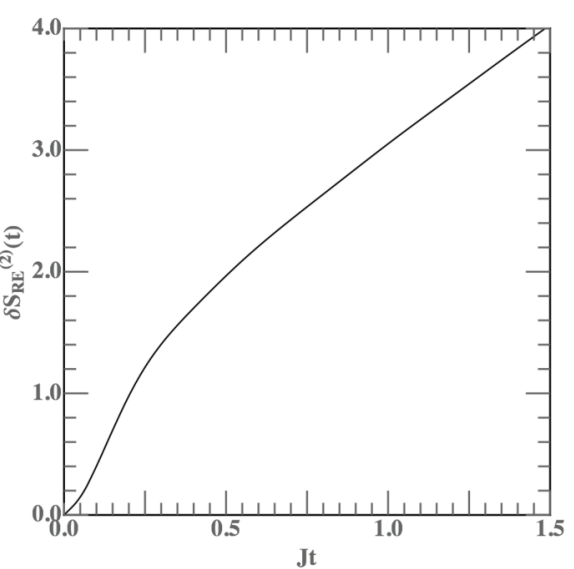

(b)

Figure 4. Heating of the "Black Hole ". The figure shows the Rényi entropy response of $\mathrm{SYK}_{4}$ with $\mathcal{J}=4$ and $\beta=10$ coupling to $\mathrm{SYK}_{2}$ model with $\mathcal{J}^{\prime}=8$ and $\beta_{\mathrm{E}}=2$. A short time $t^{2}$ growth and a long time $t$ linear growth are shown.

\subsection{Heating and evaporation with non-Markovian environment}

Here we assume the environment's hamiltonian is

$$
\hat{H}_{\mathrm{E}}=\sum_{\alpha=1}^{M} \sum_{j k=1}^{N}\left(J_{j k, \alpha L}^{\prime}-\mu\right) \hat{\psi}_{j, \alpha L}^{\dagger} \hat{\psi}_{k, \alpha L}+\sum_{\alpha=1}^{M} \sum_{j k=1}^{N}\left(J_{j k, \alpha R}^{\prime}+\mu\right) \hat{\psi}_{j, \alpha R}^{\dagger} \hat{\psi}_{k, \alpha R},
$$

where

$$
\overline{J_{j k, \alpha L(R)}^{\prime}}=0, \quad \overline{\left(J_{j k, \alpha L(R)}^{\prime}\right)^{2}}=\frac{1}{4 N}\left(\mathcal{J}^{\prime}\right)^{2} .
$$

The interactions between the system and the environment is

$$
\hat{V}=g \sum_{j=1}^{N} \sum_{\alpha=1}^{M} \sum_{\sigma=L, R}\left(\hat{\chi}_{j} \hat{\psi}_{j, \alpha \sigma}^{\dagger}+\hat{\chi}_{j} \hat{\psi}_{j, \alpha \sigma}\right)
$$

For our problem, this model change is equivalent to a simple change in environment's spectral function.

$$
\mathcal{A}_{\xi}\left(\omega, \beta_{\mathrm{E}}\right)=\left(\sqrt{1-\frac{(\omega-\mu)^{2}}{\mathcal{J}^{\prime 2}}}+\sqrt{1-\frac{(\omega+\mu)^{2}}{\mathcal{J}^{\prime 2}}}\right)
$$

Here we assume $\mu>\mathcal{J}^{\prime}$, such that we have a gapped system. Here we take $\mathcal{J}^{\prime}=0.5, \mu=1$. Again we apply eq. (2.20), then we show the numerical results in figure 5. We compared the results with the same parameter $\mathcal{J}^{\prime}$ with the previous spectral function. For a case where $\beta_{\mathrm{E}}<\beta$, the presence of a gap can speed up the entanglement entropy decreasing.

A very non-trivial and interesting dynamical behavior is observed that the entropy experiences an oscillation before a late time linear growth. This situation happens when the environment temperature is close to the system's temperature. We find the gap in 


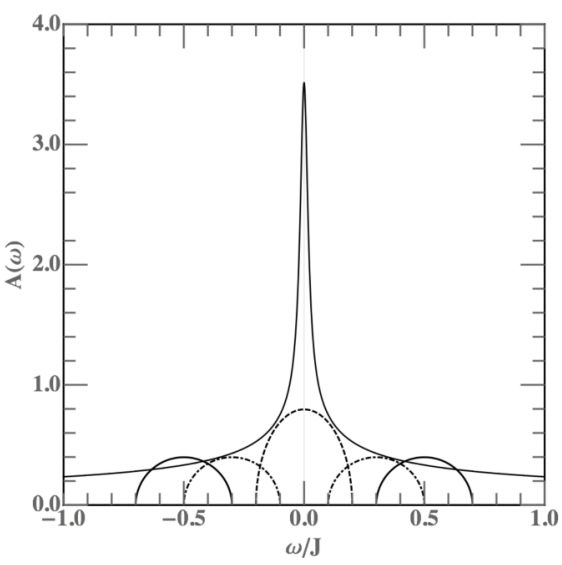

(a)

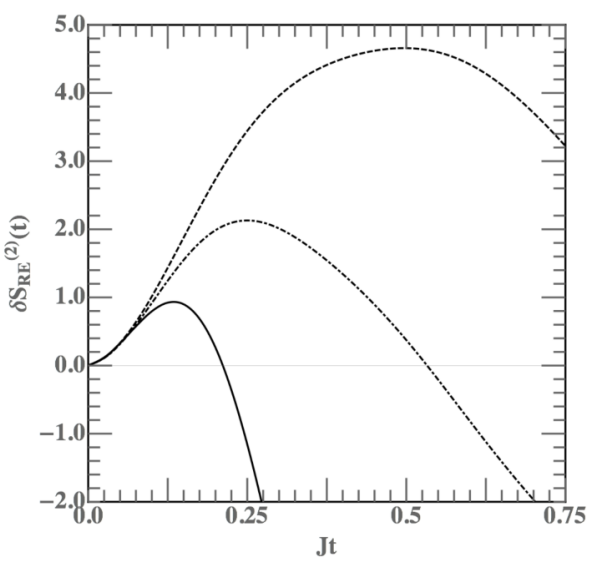

(b)

Figure 5. Here we take $\mathcal{J}=4, \beta=10, \mathcal{J}^{\prime}=0.8$. In (a) we give the spectral function of the system and the environment. The dashed line is for $\mu=0$., dot-dashed line is for $\mu=1.2$ and the solid line is for $\mu=2$. The gap of the spectrum is increasing from 0 to 1.2. In (b) we show the Rényi entropy response for $\beta_{\mathrm{E}}=2, \mu=0$. as dashed line; $\mu=1.2$ case as dot-dashed line and $\mu=2$. as solid line.

the spectrum can result in a non-trivial memory effect in entropy dynamics where the heat flow direction can change at least twice. Deeper reasons for the extra oscillation behavior in RE dynamics remain to be further understood. By comparing the behavior of the gapless spectrum and the gapped spectrum under similar circumstance, we find the gapped spectrum bring the oscillation of RE to shorter time scale.

\section{Conclusion}

In summary, we construct a perturbative method for calculating the Rényi entropy for a sudden coupling between a system and an environment. On condition that the coupling between two systems is linear coupling, we obtained a general formula for Rényi entropy response for bosonic systems and fermionic systems respectively. One can find the entropy response is related to the spectral function of the system and the environment as well as a distribution kernel function $\mathcal{W}^{B, F}$. We find the short time behavior of Rényi entropy response follows a $t^{2}$ law, and later it follows a linear $t$ law. Fermi statistics can result in a non-monotonic behavior in Rényi entropy dynamics which may have a gravitational explanation as a Page curve in black evaporation. Further, among two reasons for a bath being non-Markovian, we find the distribution kernel plays a vital role in the behavior of entanglement entropy dynamics, while the spectral function of the environment controls the detailed behavior of the entropy dynamics. Further understanding of these perturbative results in gravity language, as well as the back action of the environment to the sudden coupling of the system, are left for future study.

At the closing stage of this paper, we notice a similar paper on this topic as arXiv:2011.09622 by Dadras and Kitaev [46]. 


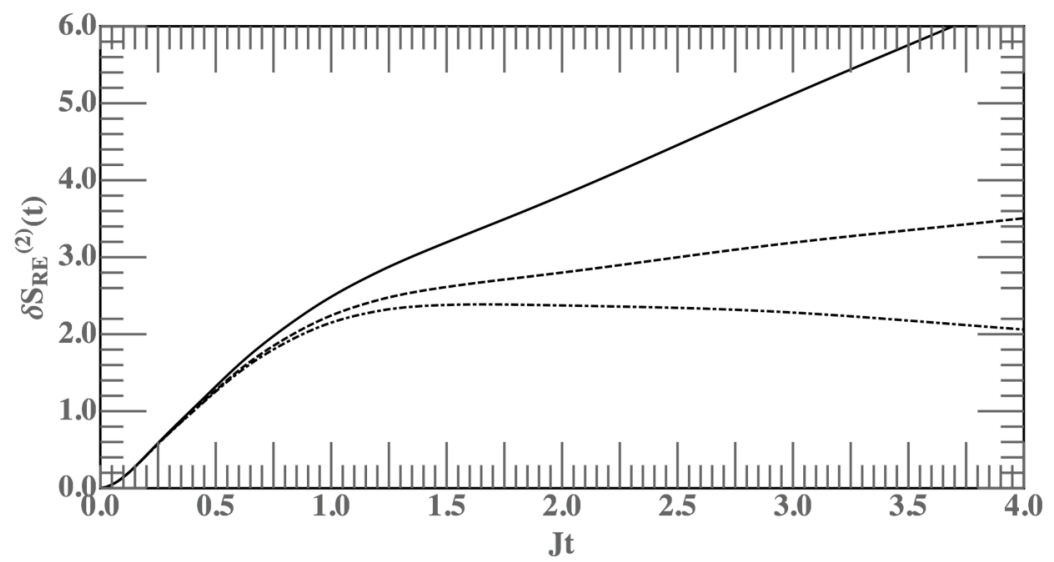

(a)

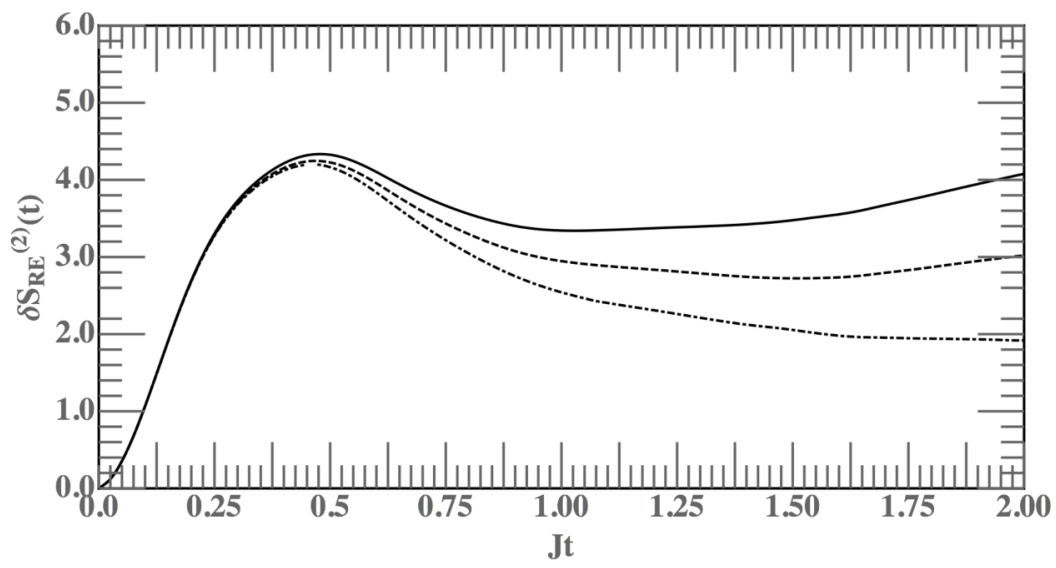

(b)

Figure 6. In (a) we show the RE response for environment with gapless spectrum, $\beta=10, \mathcal{J}=4$, $\beta_{\mathrm{E}}=4.5,8$ and 12 respectively with solid line, dashed line and dot-dashed line, $\mathcal{J}^{\prime}=0.8$; in (b) we show an oscillation behavior of $\delta S_{\mathrm{RE}}^{(2)}$ when $\beta_{\mathrm{E}} \approx \beta$. Here $\beta$ is fixed as 10 . Here the solid line has a temperature $\beta_{\mathrm{E}}=4.5$. The dashed line is at the temperature $\beta_{\mathrm{E}}=8$ and the dot-dashed line is at the environment temperature $\beta_{\mathrm{E}}=12$.

\section{Acknowledgments}

We thank Pengfei Zhang, Yiming Chen and Hui Zhai for discussions. This work is supported by NSFC under Grant No. 11734010, Beijing Natural Science Foundation (Z180013). 
Open Access. This article is distributed under the terms of the Creative Commons Attribution License (CC-BY 4.0), which permits any use, distribution and reproduction in any medium, provided the original author(s) and source are credited.

\section{References}

[1] G.D. Mahan, Many Particle Physics, Plenum Press, New York and London (1981).

[2] L. Pan, X. Chen, Y. Chen and H. Zhai, Non-Hermitian Linear Response Theory, Nat. Phys. 16 (2020) 767.

[3] R. Bouganne et al., Anomalous decay of coherence in a dissipative many-body system, Nat. Phys. 16 (2020) 21.

[4] J.M. Deutsch, Quantum statistical mechanics in a closed system, Phys. Rev. A 43 (1991) 2046.

[5] M. Srednicki, Chaos and quantum thermalization, Phys. Rev. E 50 (1994) 888.

[6] P. Calabrese and J.L. Cardy, Entanglement entropy and quantum field theory, J. Stat. Mech. 0406 (2004) P06002 [hep-th/0405152] [INSPIRE].

[7] J.R. Garrison and T. Grover, Does a single eigenstate encode the full Hamiltonian?, Phys. Rev. X 8 (2018) 021026 [arXiv:1503.00729] [INSPIRE].

[8] P. Hosur, X.-L. Qi, D.A. Roberts and B. Yoshida, Chaos in quantum channels, JHEP 02 (2016) 004 [arXiv: 1511.04021] [INSPIRE].

[9] R. Fan, P. Zhang, H. Shen and H. Zhai, Out-of-Time-Order Correlation for Many-Body Localization, Sci. Bull. 62 (2017) 707 [arXiv: 1608.01914] [INSPIRE].

[10] M. Rangamani and T. Takayanagi, Holographic Entanglement Entropy, Springer (2017) DOI.

[11] J.M. Maldacena, The large- $N$ limit of superconformal field theories and supergravity, Int. J. Theor. Phys. 38 (1999) 1113 [hep-th/9711200] [INSPIRE].

[12] S. Ryu and T. Takayanagi, Holographic derivation of entanglement entropy from AdS/CFT, Phys. Rev. Lett. 96 (2006) 181602 [hep-th/0603001] [InSPIRE].

[13] V.E. Hubeny, M. Rangamani and T. Takayanagi, A Covariant holographic entanglement entropy proposal, JHEP 07 (2007) 062 [arXiv:0705.0016] [INSPIRE].

[14] N. Engelhardt and A.C. Wall, Quantum Extremal Surfaces: Holographic Entanglement Entropy beyond the Classical Regime, JHEP 01 (2015) 073 [arXiv:1408.3203] [INSPIRE].

[15] R. Islam et al., Measuring entanglement entropy in a quantum many-body system, Nature 528 (2015) 77.

[16] A.M. Kaufman, M.E. Tai, A. Lukin, M. Rispoli, R. Schittko, P.M. Preiss and M. Greiner, Quantum thermalization through entanglement in an isolated many-body system, Science $\mathbf{3 5 3}$ (2016) 6301.

[17] J. Li et al., Measuring Out-of-Time-Order Correlators on a Nuclear Magnetic Resonance Quantum Simulator, Phys. Rev. X 7 (2017) 031011 [arXiv: 1609.01246] [INSPIRE].

[18] A. Lukin, M. Rispoli, R. Schittko, M.E. Tai, A.M. Kaufman, S. Choi, V. Khemani, J. Léonard and M. Greiner, Probing entanglement in a many-body-localized system, Science $\mathbf{3 6 4}$ (2019) 256 [arXiv:1805.09819v2]. 
[19] T. Brydges, A. Elben, P. Jurcevic, B. Vermersch, C. Maier, B.P. Lanyon, P. Zoller, R. Blatt, C.F. Roos, Probing Rényi entanglement entropy via randomized measurements, Science $\mathbf{3 6 4}$ (2019) 260.

[20] A. Kitaev, A simple model of quantum holography (part 1), talk at KITP, April 7, 2015, http://online.kitp.ucsb.edu/online/entangled15/kitaev/.

[21] A. Kitaev, A simple model of quantum holography (part 2), talk at KITP, May 27, 2015, http://online.kitp.ucsb.edu/online/entangled15/kitaev2/.

[22] S. Sachdev and J. Ye, Gapless spin fluid ground state in a random, quantum Heisenberg magnet, Phys. Rev. Lett. 70 (1993) 3339 [cond-mat/9212030] [INSPIRE].

[23] J. Maldacena and D. Stanford, Remarks on the Sachdev-Ye-Kitaev model, Phys. Rev. D 94 (2016) 106002 [arXiv:1604.07818] [INSPIRE].

[24] A. Kitaev and S.J. Suh, The soft mode in the Sachdev-Ye-Kitaev model and its gravity dual, JHEP 05 (2018) 183 [arXiv:1711.08467] [INSPIRE].

[25] J. Maldacena, D. Stanford and Z. Yang, Conformal symmetry and its breaking in two dimensional Nearly Anti-de-Sitter space, PTEP 2016 (2016) 12C104 [arXiv:1606.01857] [INSPIRE].

[26] D.J. Gross and V. Rosenhaus, The Bulk Dual of SYK: Cubic Couplings, JHEP 05 (2017) 092 [arXiv: 1702.08016] [INSPIRE].

[27] R. Jackiw, Lower Dimensional Gravity, Nucl. Phys. B 252 (1985) 343 [InSPIRE].

[28] C. Teitelboim, Gravitation and Hamiltonian Structure in Two Space-Time Dimensions, Phys. Lett. B 126 (1983) 41 [INSPIRE].

[29] Y. Gu, A. Lucas and X.-L. Qi, Spread of entanglement in a Sachdev-Ye-Kitaev chain, JHEP 09 (2017) 120 [arXiv:1708.00871] [INSPIRE].

[30] Y. Huang and Y. Gu, Eigenstate entanglement in the Sachdev-Ye-Kitaev model, Phys. Rev. D 100 (2017) 041901 [arXiv: 1709.09160] [INSPIRE].

[31] C. Liu, X. Chen and L. Balents, Quantum Entanglement of the Sachdev-Ye-Kitaev Models, Phys. Rev. B 97 (2018) 245126 [arXiv:1709.06259] [InSPIRE].

[32] P. Zhang, Evaporation dynamics of the Sachdev-Ye-Kitaev model, Phys. Rev. B 100 (2019) 245104 [arXiv: 1909.10637] [INSPIRE].

[33] Y. Chen, X.-L. Qi and P. Zhang, Replica wormhole and information retrieval in the SYK model coupled to Majorana chains, JHEP 06 (2020) 121 [arXiv: 2003.13147] [INSPIRE].

[34] P. Zhang, C. Liu and X. Chen, Subsystem Rényi Entropy of Thermal Ensembles for SYK-like models, SciPost Phys. 8 (2020) 094 [arXiv:2003.09766] [InSPIRE].

[35] X.-L. Qi and P. Zhang, The Coupled SYK model at Finite Temperature, JHEP 05 (2020) 129 [arXiv: 2003.03916] [INSPIRE].

[36] A. Haldar, S. Bera and S. Banerjee, Rényi entanglement entropy of Fermi and non-Fermi liquids: Sachdev-Ye-Kitaev model and dynamical mean field theories, Phys. Rev. Res. 2 (2020) 033505 [arXiv:2004.04751] [INSPIRE].

[37] P. Zhang, Entanglement Entropy and its Quench Dynamics for Pure States of the Sachdev-Ye-Kitaev model, JHEP 06 (2020) 143 [arXiv: 2004.05339] [INSPIRE].

[38] J. Maldacena and X.-L. Qi, Eternal traversable wormhole, arXiv:1804.00491 [INSPIRE]. 
[39] G. Penington, Entanglement Wedge Reconstruction and the Information Paradox, JHEP 09 (2020) 002 [arXiv: 1905.08255] [INSPIRE].

[40] A. Almheiri, N. Engelhardt, D. Marolf and H. Maxfield, The entropy of bulk quantum fields and the entanglement wedge of an evaporating black hole, JHEP 12 (2019) 063 [arXiv: 1905.08762] [INSPIRE].

[41] A. Almheiri, R. Mahajan, J. Maldacena and Y. Zhao, The Page curve of Hawking radiation from semiclassical geometry, JHEP 03 (2020) 149 [arXiv: 1908.10996] [INSPIRE].

[42] S.F. Lokhande, G.W.J. Oling and J.F. Pedraza, Linear response of entanglement entropy from holography, JHEP 10 (2017) 104 [arXiv: 1705.10324] [INSPIRE].

[43] Y. Chen and P. Zhang, Entanglement Entropy of Two Coupled SYK Models and Eternal Traversable Wormhole, JHEP 07 (2019) 033 [arXiv: 1903.10532] [INSPIRE].

[44] D.N. Page, Information in black hole radiation, Phys. Rev. Lett. 71 (1993) 3743 [hep-th/9306083] [INSPIRE].

[45] D.N. Page, Time Dependence of Hawking Radiation Entropy, JCAP 09 (2013) 028 [arXiv: 1301.4995] [INSPIRE].

[46] P. Dadras and A. Kitaev, Perturbative calculations of entanglement entropy, JHEP 03 (2021) 198 [arXiv: 2011.09622] [INSPIRE]. 